Multicarrier DS-CDMA:

\title{
A Multiple Access Scheme for Ubiquitous Broadband Wireless Communications
}

\author{
Lie-Liang Yang and Lajos Hanzo, University of Southampton
}

\begin{abstract}
In this article we identify some of the key problems that may be encountered when designing a broadband multiple access system with bandwidth on the order of tens or even hundreds of megahertz. We commence with a comparative discussion in terms of the characteristics of three typical code-division multiple access schemes: single-carrier directsequence CDMA (SC DS-CDMA), multicarrier CDMA (MC-CDMA), and multicarrier DSCDMA (MC DS-CDMA). Specifically, their benefits and deficiencies are analyzed when aiming to support ubiquitous communications over a variety of channels encountered in indoor, open rural, suburban, and urban environments. It is shown that when communicating in such diverse environments, both SC DSCDMA and MC-CDMA exhibit certain limitations that are hard to circumvent. By contrast, when appropriately selecting the system parameters and using transmit diversity, MC DSCDMA becomes capable of adapting to such diverse propagation environments at a reasonable detection complexity.
\end{abstract}

\section{INTRODUCTION}

Future generations of broadband wireless mobile systems will aim to support a wide range of services and bit rates by employing a variety of techniques capable of achieving the highest possible spectrum efficiency [1]. Code-division multiple access (CDMA) schemes have been considered as attractive multiple access schemes in both second-generation (2G) and third-generation $(3 \mathrm{G})$ wireless systems. Recently interest in wireless communications has been shifting in the direction of broadband systems $[1,2]$. This is mainly due to the expected spread of the wireless Internet and the continued dramatic increase in demand for all types of advanced wireless multimedia services, including conventional voice and data transmissions. Furthermore, future generations of broadband wireless systems are expected to support ubiquitous communications, regardless of the propagation environment encountered, while maintaining the required quality of service (QoS).

In the context of broadband wireless communications using CDMA without the assistance of frequency/time hopping, the main multiple access options include single-carrier directsequence CDMA (SC DS-CDMA) using timedomain (T-domain) DS spreading [1], multicarrier CDMA (MC-CDMA) using frequency-domain (F-domain) spreading [1, 3], as well as multicarrier DS-CDMA (MC DSCDMA) using T-domain DS spreading of the individual subcarrier signals $[1,3]$. The spectrum as well as the spreading model of the above three CDMA arrangements are shown in Figs. 1, 2 , and 3, all of which have approximately the same bandwidth. This figure will be augmented in more depth in the next section.

In this article we investigate the behavior of the above three CDMA schemes when communicating over broadband wireless channels. We will show that regardless of the communication environments encountered, both SC DS-CDMA and MC-CDMA exhibit more severe problems than MC DS-CDMA. Broadband MC DS-CDMA augmented by transmit diversity is capable of mitigating the problems imposed by broadband wireless channels. It is shown that by appropriately selecting the system parameters, transmit diversity assisted broadband MC DS-CDMA is capable of supporting wireless communications in diverse propagation environments. Furthermore, in this article the issue of capacity improvement achievable by broadband MC DS-CDMA systems is also investigated. Let us first embark on a rudimentary overview of the above three CDMA schemes. 


\section{AN OVERVIEW OF CDMA SCHEMES USING No FREQUENCY/TIME HOPPING}

Hara and Prasad have provided an excellent overview of both SC DS-CDMA and the different multicarrier CDMA schemes in [3]. In this section we provide a different digest of SC DSCDMA, MC-CDMA, and MC DS-CDMA, with specific emphasis on the transmitted signals' structures. As discussed in [3], MC-CDMA using DS-spread subcarrier signals can be further divided into multitone DS-CDMA, orthogonal MC DS-CDMA, and MC DS-CDMA using no subcarrier overlapping. The authors of this contribution have shown in [4] that the above three types of MC DS-CDMA schemes using a specific frequency spacing between two adjacent subcarriers can be unified in the family of generalized MC DS-CDMA schemes using an arbitrary frequency spacing, $\Delta$, between two adjacent subcarriers. In this article we take orthogonal MC DS-CDMA as an example and refer to it simply as MC DS-CDMA, unless it would cause confusion. However, our analysis can readily be extended to MC DS-CDMA schemes using various frequency spacing values of $\Delta$.

\section{SC DS-CDMA}

By definition, an SC DS-CDMA scheme transmits DS-spread signals using a single carrier. In SC DS-CDMA the original data stream is spread using a given spreading code in the T-domain, as shown in Fig. 1. Hence, the transmitted signal in SC DS-CDMA using binary phase shift keying (BPSK) modulation can be expressed as

$$
\begin{aligned}
s_{D S}(t)= & \sqrt{2 P} \sum_{i=-M}^{M} \sum_{j=0}^{N-1} b[i] c[j] \\
& p_{T_{c l}}\left(t-i T_{b}-j T_{c l}\right) \cos \left(2 \pi f_{c} t\right),
\end{aligned}
$$

where $P$ and $f_{c}$ represent the transmitted power and carrier frequency, respectively, $T_{b}$ and $T_{c 1}$ represent the bit duration and chip duration, respectively, and the processing gain (spreading factor) of $N=T_{b} / T_{c 1}$ represents the number of chips per bit. Furthermore, in Eq. $12 M+1$ represents the number of bits conveyed by a transmitted data burst, $b[i] \in\{+1,-1\}$ is the $i$ th transmitted bit, while $c[j] \in\{+1,-1\}$ is the $j$ th chip of the spreading code, and finally, $p_{\tau}(t)$ represents the chip waveform defined over the interval $[0, \tau)$.

The number of users supported by SC DSCDMA depends on the achievable processing gain and the cross-correlation characteristics of the spreading codes. When communicating over frequency-selective fading channels, the number of users supported by SC DS-CDMA is also influenced by the auto-correlation characteristics of the spreading codes.

\section{MC-CDMA}

MC-CDMA conveys the transmitted signals using a number of subcarriers. In MC-CDMA the transmitter spreads each parallel substream of data generated with the aid of serial-to-paral-

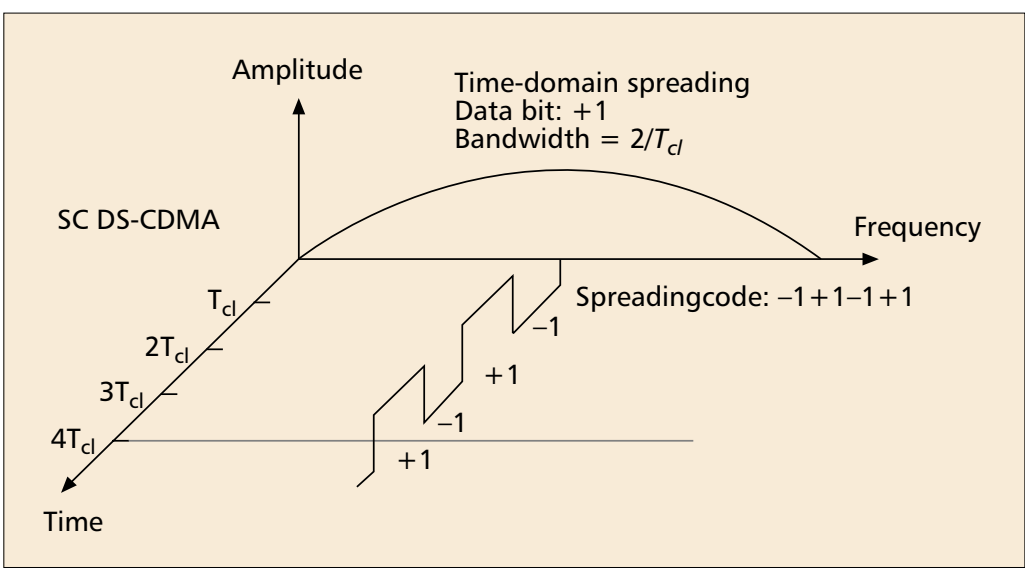

Figure 1. Power spectra and time-domain signal waveforms associated with single-carrier DS-CDMA (SCDS-CDMA) using the time-domain spreading code $\{-1+1-1+1\}$

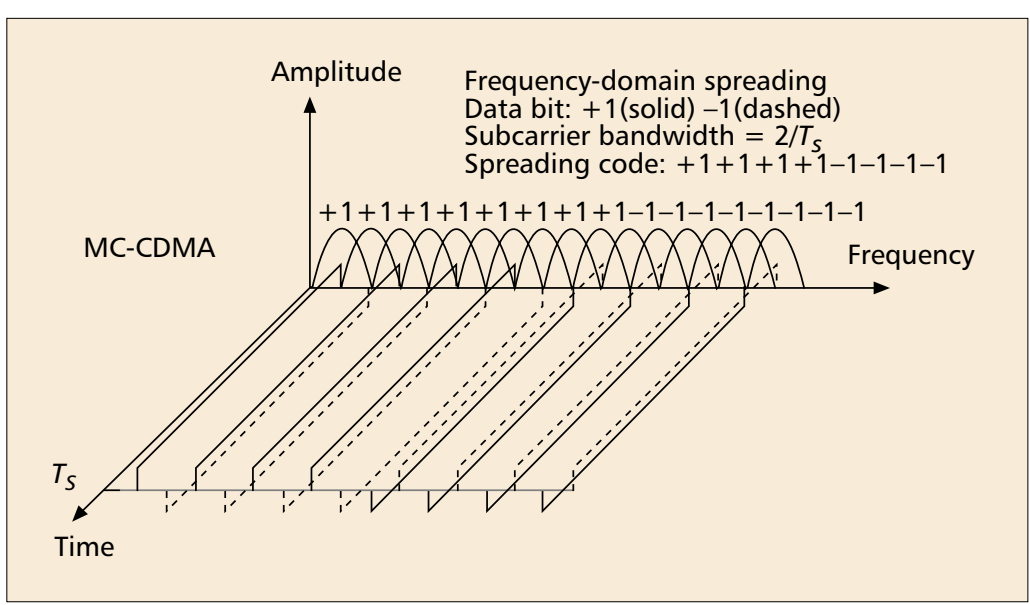

Figure 2. Power spectra and time-domain signal waveforms associated with $M C-C D M A$ using the frequency-domain spreading code $\{+1+1+1+1-1$ $-1-1-1\}$ and $S-P$ conversion associated with $\mathrm{U}=2$ bits.

lel (S-P) conversion across $N_{p}$ number of subcarriers using a given $N_{p}$-chip spreading code, $\{c[0]$, $\left.c[1], \ldots, c\left[N_{p}-1\right]\right\}[3]$. As seen in Fig. 2, the transmitted MC-CDMA signal using BPSK modulation can be expressed as

$$
\begin{aligned}
s_{M C}(t) & =\sqrt{\frac{2 P}{U N_{p}}} \sum_{i=-M}^{M} \sum_{u=0}^{U-1} \sum_{j=0}^{N_{p}-1} b_{i}[u] c[j] \\
& p_{T_{s}}\left(t-i T_{s}\right) \cos \left[2 \pi\left(f_{c}+F_{j U+u}\right) t\right],
\end{aligned}
$$

where $P, c[j], f_{c}$ and $p_{t}(t)$ have the same meaning as in Eq. 1. In Eq. $2 U$ represents the number of bits that are S-P converted, where each transmitted symbol contains $U$ data bits, $2 M+1$ represents the number of $U$-bit symbols conveyed by a transmitted data burst, and $b_{i}[u] \in\{+1,-1\}$ represents the $u$ th bit of the $i$ th transmitted symbol. As seen in Eq. 2, the total number of subcarriers is $Q=U N_{p}$. Let $f_{0}<f_{1}<\ldots<f_{U N p-1}$. Then the $N_{p}$ number of subcarriers $\left\{f_{j U+u}\right\}_{j=0} N_{p-1}$ having the maximum possible frequency spacing between any two subcarriers are used for conveying the $N_{p}$ chips of the substream $b_{i}[u]$ for $u$ $=0,1, \ldots, U-1$. Furthermore, in Eq. $2 T_{s}$ represents both symbol duration and chip duration, 


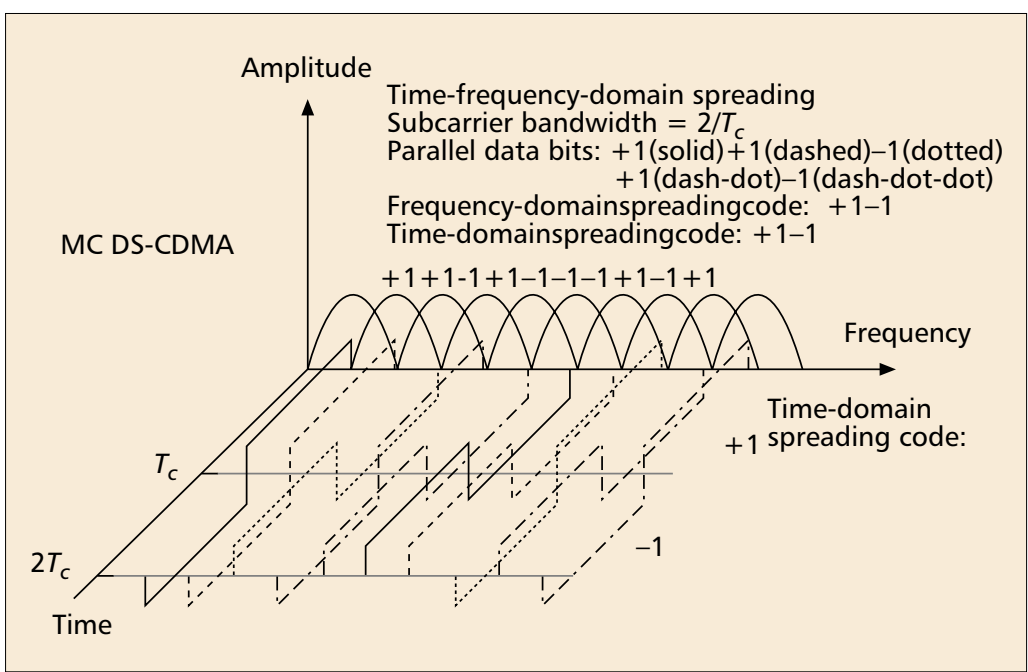

Figure 3. Power spectra and time-domain signal waveforms associated with $M C D S-C D M A$ using the time-domain spreading code $\{+1-1\}$, the frequency-domain spreading code $\{+1-1\}$, and $S-P$ conversion associated with $\mathrm{U}=$ 5 bits.

${ }^{1}$ This assumption may be satisfied by appropriately choosing the number of subcarriers occupying the bandwidth available, provided that the channel's impulse response and the corresponding channel transfer functions are known. since in MC-CDMA the symbol duration and chip duration assume the same value.

The number of users supported by MCCDMA depends on both the processing gain and the cross-correlation characteristics of the different spreading codes. However, since in MCCDMA each subcarrier signal is assumed to be experiencing flat fading, ${ }^{1}$ no multipath-induced intersymbol interference (ISI) is imposed on the subcarrier signals, which would impair their autocorrelation. Hence, the number of users supported by MC-CDMA remains independent of the auto-correlation characteristics of the spreading codes. If, however, the MC-CDMA system's transmission bit rate is high [3], the transmitted signal would experience frequencyselective fading. In this case $\mathrm{S}-\mathrm{P}$ conversion invoking a high number of input data bits and a high number of subcarriers can be employed to prevent the subcarriers from experiencing frequency-selective fading.

\section{DS-CDMA}

Generally, MC DS-CDMA transmits T-domain DS-spread signals using multiple subcarriers [5]. The philosophy of the MC DS-CDMA scheme considered here is more general, as shown in Fig. 3, which employs F-domain spreading in addition to T-domain spreading (i.e., employs TF-domain spreading). The corresponding MC DS-CDMA transmitter scheme includes an S-P converter, which reduces the subcarrier data rate by mapping the serial data to a number of reduced-rate parallel streams. In MC DS-CDMA systems DS-based T-domain subcarrier spreading gain associated with each subcarrier signal, while F-domain spreading across several subcarriers is employed to further increase the total attainable processing gain. The total processing gain is usually determined by the product of the T-domain and F-domain spreading factors. Following the philosophy of Fig. 3, the transmitted MC DS-CDMA signal using BPSK modulation can be expressed as ing is invoked to increase the achievable process-

$$
\begin{aligned}
s_{M D S}(t) & =\sqrt{\frac{2 P}{U S}} \sum_{i=-M}^{M} \sum_{u=0}^{U-1} \sum_{s=0}^{S-1} \sum_{j=0}^{N_{p}-1} b_{i}[u] c_{t}[j] \\
p_{T_{c}} & \left(t-i T_{s}-j T_{c}\right) c_{f}[s] \cos \left[2 \pi\left(f_{c}+F_{s} U+u\right) t\right],
\end{aligned}
$$

where $P, b_{i}[u], f_{c}$ and $p_{\tau}(t)$ have the same interpretation as in Eq. $2, c_{t}[j]$ represents the $j$ th chip of the T-domain spreading code, while $c_{f}[s]$ represents the $s$ th chip of the F-domain spreading code. The $Q=U S$ number of subcarrier frequencies are represented by $\left\{f_{s U+u}\right\}$ for $u=0,1$, $\ldots, U-1 ; s=0,1, \ldots, S-1$. As in Eq. 2, formulated in the context of MC-CDMA, $S$ number of subcarriers are used for F-domain spreading of the same data bit, and we have the maximum possible frequency spacing between any two of the $S$ subcarriers. Furthermore, in Eq. $3(2 M+$ 1 ) is the total number of symbols transmitted during a transmission burst, and the MC DSCDMA signal has a symbol duration of $T_{s}$ as well as a chip duration of $T_{c}$.

Similar to SC DS-CDMA but in contrast to MC-CDMA, the DS spread subcarrier signals of MC DS-CDMA may experience frequency-selective fading. Hence, the total number of users supported by MC DS-CDMA is jointly determined by the T-domain and F-domain spreading factors, as well as by the auto-correlation and cross-correlation characteristics of the spreading codes employed.

In conventional MC DS-CDMA [3, 5], which employs no F-domain spreading, the chips of a DS spread subcarrier signal can be repeated across $S$ number of subcarriers, which is referred to here as $F$-domain repetition, in order to achieve a higher grade of frequency diversity. Instead of solely using F-domain repetition, the transmitted data stream can be spread in both the T-domain and F-domain (i.e., in TF-domain) as indicated in Fig. 3 and Eq. 3. As will be shown later, the employment of TF-domain spreading will allow us to mitigate the deficiency that the number of users supported by MC DS-CDMA decreases on increasing repetition depth $S$.

\section{FLEXIBILITY COMPARISON}

Above we have reviewed three typical CDMA schemes: SC DS-CDMA, MC-CDMA and MC DS-CDMA. In the context of broadband wireless communications the design and reconfiguration flexibilities constitute important considerations. The flexibility of a multiple access scheme depends on its degree of freedom, which is defined as the number of independent parameters that can be controlled and adapted during the system design phase or reconfigured during the communications session with the aid of advanced techniques facilitated by the concept of software radios. Let us assume that the above three typical CDMA schemes employ a given zero-to-zero system bandwidth of $W_{s}=$ $2 / T_{c 1}$. Furthermore, they use a common chip waveform and employ the same BPSK data modulation scheme. We also assume that these CDMA systems support a common data rate of $R_{b}=1 / T_{b}$. Then, in addition to the aforementioned degrees of freedom, the range of other parameters that can be reconfigured by the CDMA schemes considered are as follows: 
- In the context of SC DS-CDMA no other degrees of freedom are available. In other words, the characteristics of the transmitted signal in SC DS-CDMA are fully determined by the above mentioned degrees of freedom.

- In the context of MC-CDMA, another degree of freedom is the number of bits, $U$, involved in the S-P conversion. This parameter determines both the symbol duration and chip duration, which are expressed as $T_{s}=T_{c}=U T_{b}$. It also determines the total number of subcarriers within the bandwidth of $W_{s}=2 / T_{c 1}$, which can be expressed as $Q$ $=\left(2 U T_{b} / T_{c 1}-1\right)$.

- In MC DS-CDMA systems there are another three degrees of freedom in addition to the above mentioned ones. The first is the chip duration, $T_{c}$, which determines the total number of subcarriers $Q$, yielding, for example, $Q=\left(2 T_{c} / T_{c 1}-1\right)$ for orthogonal $\mathrm{MC}$ DS-CDMA. The second is the number of bits, $U$, involved in the S-P conversion, which determines the symbol duration $\left(T_{s}=U T_{b}\right)$. Furthermore, the above two degrees of freedom determine the spreading factor of each subcarrier signal, which can be expressed as $N=U T_{b} / T_{c}$. They also determine the Fdomain repetition depth, $S$, or the F-domain spreading codes' length, $S$, which can be expressed as $S=Q / U$. Finally, the third parameter associated with MC DS-CDMA is the frequency spacing $\Delta$ between two adjacent subcarriers [4], which may assume a value spanning the range from $1 / T_{s}$ to $2 / T_{c}$. As shown in [4], in MC DS-CDMA systems the system's total transmission bandwidth $W_{s}$, total number of subcarriers $Q=U S$, subcarrier spacing $\Delta$, and chip duration $T_{c}$ of the T-domain spreading codes obey the relationship of $W_{s}=(U S-1) \Delta+2 / T_{c}$. MC DS-CDMA using an arbitrary spacing value of $\Delta$ includes the subclasses of multitone DS-CDMA [6] using $\Delta=1 / T_{s}$ and orthogonal MC DS-CDMA [5] opting for $\Delta=1 / T_{c}$ as special cases. The results of [4] show that the parameter $\Delta$ can be optimized to enhance the MC DS-CDMA system's bit error rate (BER) performance.

\section{BROADBAND WIRELESS COMMUNICATIONS BASED ON CDMA}

\section{Characteristics of Broadband Channels}

Future generations of broadband wireless systems will aim to support a wide range of services and bit rates in a bandwidth on the order of tens or even hundreds of megahertz. Thus, these broadband wireless signals may have significantly higher bandwidth than the coherence bandwidth of the channels encountered, and so will inevitably experience severe frequency-selective fading. Furthermore, broadband wireless systems (using, e.g., multicarrier transmissions) may encounter a different Doppler frequency shift for the lowest and highest subcarriers, due to the high frequency difference between them.

\section{LIMITATIONS OF \\ BROADBAND SC DS-CDMA AND BROADBAND MC-CDMA}

In Table 1 we summarized some of the typical signal characteristics and the corresponding receiver models in the context of the SC DSCDMA, MC-CDMA and conventional non-Fdomain spread MC DS-CDMA systems communicating over time-varying wireless communication channels. When aiming to support broadband transmissions reaching a bandwidth of, say, $20 \mathrm{MHz}$ in diverse propagation environments, both SC DS-CDMA and MC-CDMA exhibit certain deficiencies. Specifically, the broadband wireless communication systems, for example, may have a $20 \mathrm{MHz}$ bandwidth and be required to support a bit rate of $1 \mathrm{Mb} / \mathrm{s}$. In this context both broadband SC DS-CDMA and MC-CDMA systems would experience the following problems.

Communications in diverse propagation environments cannot be readily supported by SC DSCDMA or MC-CDMA. Assuming binary transmissions (e.g., BPSK modulation), in SC DS-CDMA the transmitted symbol's duration and the data bit's duration are the same in both of the above schemes. Propagation measurements conducted in typical wireless environments including indoor, open rural, suburban, and urban areas show that the delay spread is typically distributed over the range of $[0.1 \mu \mathrm{s}, 3$ $\mu \mathrm{s}$ ] [7]. Hence, when communicating at $1 \mathrm{Mb} / \mathrm{s}$, SC DS-CDMA cannot perform well in environments having a delay spread higher than $1 \mu \mathrm{s}$. Otherwise, severe intersymbol interference (ISI) will be imposed on the adjacent symbols due to the delayed and unresolvable paths having relative delays higher than $1 \mu \mathrm{s}$. In the context of MC-CDMA, we might argue that S-P conversion may be employed for rendering the symbol duration higher than the highest delay spread encountered (e.g., $>4 \mu \mathrm{s}$ ), which would mitigate the ISI. However, employing S-P conversion in MC-CDMA will result in an increased peak-toaverage power fluctuation [8], due to the increased symbol duration and as a result of the increased number of subcarriers. Furthermore, using S-P conversion cannot mitigate the following problem.

Frequency diversity may not be efficiently exploited in broadband SC DS-CDMA. By the same token, achieving frequency diversity in $\mathrm{MC}$ CDMA may be hampered, since significant correlation may exist between the fading envelopes of adjacent subcarriers of a broadband MCCDMA system when the frequency selectivity of the channels is low. A broadband SC DS-CDMA scheme designed using a high number of RAKE fingers for propagation environments having a high delay-spread will combine noise, if the number of resolvable paths is low, since the environment encountered exhibits a low delay spread. By contrast, a broadband SC DS-CDMA scheme designed using a low number of RAKE fingers is suitable for environments with a low delay spread. However, this scheme will waste some of the effective received signal energy
Future generations

of broadband

wireless systems

will aim to

support a wide

range of services

and bit rates in a

bandwidth on the

order of tens or

even hundreds of

megahertz. These

broadband

wireless signals

hence may have

a bandwidth

significantly

higher than the

coherence

bandwidth of the channels

encountered. 


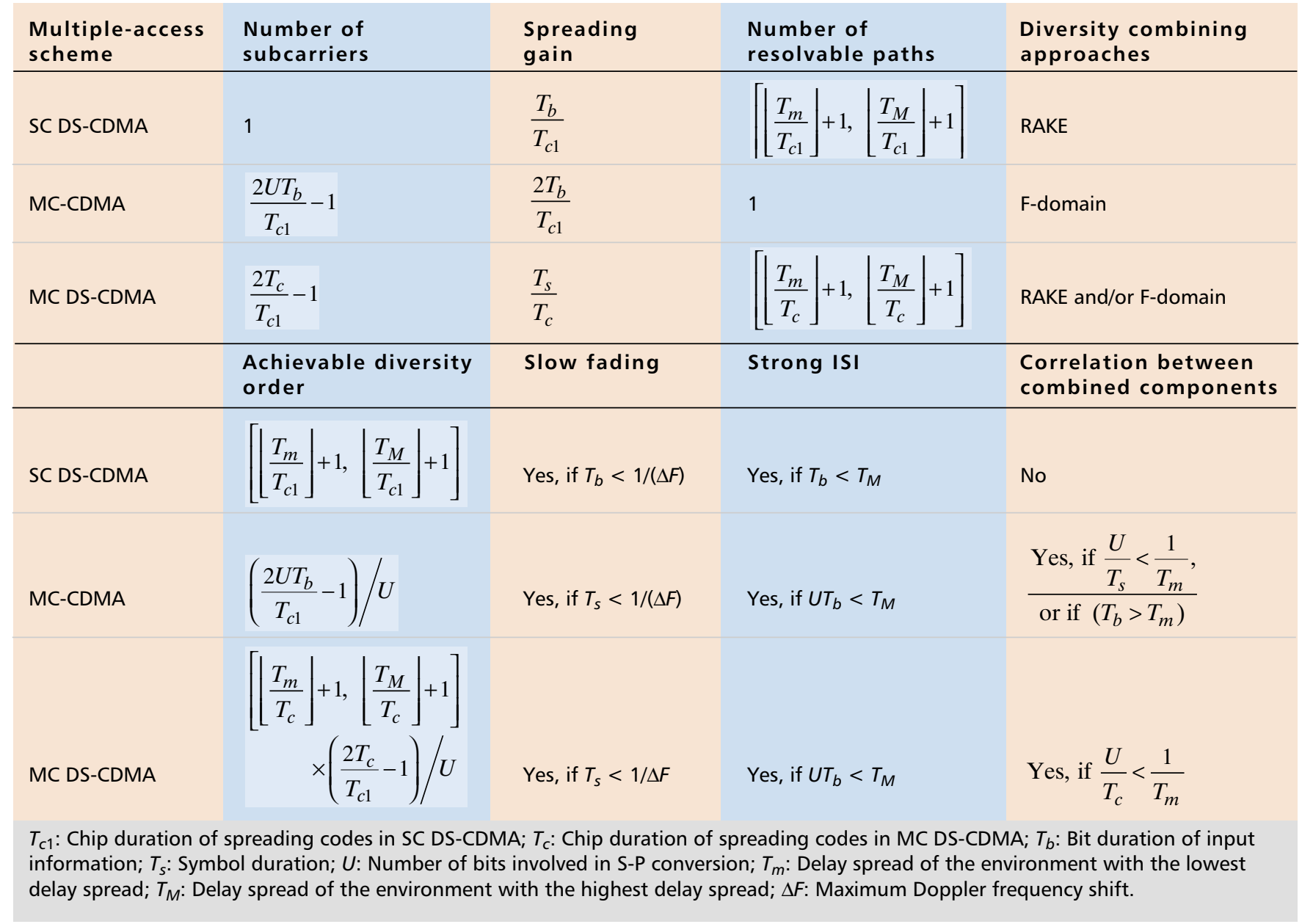

Table 1. Typical signal and receiver characteristics associated with SC DS-CDMA, MC-CDMA and conventional non-F-domain spread MC DS-CDMA communicating over wireless communications channels.

delivered by the paths that cannot be combined due to the low number of RAKE fingers in environments with a high delay spread. As a remedy, hybrid schemes based on selection combining and maximal ratio combining (SC/MRC) schemes may be employed, depending on the specific environment. In SC/MRC schemes only a fraction of the resolvable paths, the dominant ones, are selected, and then combined using MRC. However, the high complexity imposed by the estimation of all resolvable paths and the suboptimum performance due to the received signal energy loss associated with the uncombined paths are unavoidable. Consequently, a highly efficient diversity combining arrangement has to invoke an adaptive MRC scheme, which is capable of combining a time-variant number of resolvable paths encountered in various propagation environments. However, the cost of such a combining scheme is the associated increase of complexity. Again, in the context of broadband MC-CDMA the adjacent subcarriers may be exposed to correlated fading, especially if the delay spread of the channel is relatively low, resulting in the relatively high coherence bandwidth. Consequently, combining the adjacent subcarrier signals may not achieve the expected BER performance when transmitting over such low-dispersion fading channels. As indicated in Table 1, the correlation of the fading envelopes experienced by the adjacent combined subcarriers cannot be removed by S-P conversion; it can only be mitigated by employing high-latency interleaving of the transmitted data before the S$\mathrm{P}$ conversion.

Multiuser detection (MUD) is a highly efficient detection technique, which is capable of attaining near-single-user performance, while supporting a multiplicity of users and hence increasing the user capacity of wireless systems. The downlink (base-to-mobile) is expected to become the bottleneck in the forthcoming wireless Internet, which is likely to convey more downloading traffic than uplink (mobile-to-base) traffic. Moreover, the mobile handset has to be light and have relatively low signal processing complexity. Hence, at the mobile station (MS) relatively lowcomplexity detectors have to be employed. However, in order for broadband SC DS-CDMA or broadband MC-CDMA to achieve the best possible BER performance, both require high-complexity MUDs. It is well recognized that the MUD algorithms' complexity typically increases at least linearly with the total number of users detected by the system. The complexity of MUD cannot be sufficiently relaxed, even if orthogonal spreading codes and synchronous downlink transmissions are used. The orthogonality of the spreading codes used in broadband SC DS- 
CDMA will be destroyed by the delay spread of the channel, which results in multipath interference imposed by both the desired user and the other users. By contrast, the orthogonality of the spreading codes used in broadband MC-CDMA employing F-domain spreading will be destroyed by the frequency-selective fading experienced by the different subcarriers. In the context of downlink transmissions, the orthogonality of the spreading codes may be restored in the MCCDMA receiver with the aid of the zero-forcing approach conditioned on perfect channel estimation, and at the expense of noise enhancement [3]. Furthermore, the receiver of SC DS-CDMA requires signal processing at a rate comparable to the chip rate, which is extremely high in broadband SC DS-CDMA systems.

Transmit diversity [9] using multiple base station (BS) antennas has been proposed for boosting the capacity and data rate of CDMA systems. In the context of SC DS-CDMA communicating over various propagation channels including indoor, open rural, suburban, and urban areas, transmit diversity schemes designed on the basis of encountering a low number of resolvable paths or based on the premise of encountering a constant number of resolvable paths may not achieve the maximum attainable throughput. In an MC-CDMA system, usually each subcarrier signal is assumed to experience flat fading. However, the actual diversity gain achieved by using multiple BS antennas and combining the subcarrier signals is time-variant. This is because the coherence bandwidth associated with the abovementioned various communication environments is different. A desirable multiple access scheme designed for diverse propagation environments is typically expected to be able to achieve a constant diversity order and maintain similar BER performance, regardless of which communication channels are encountered.

\section{USING BROADBAND MC DS-CDMA FOR SUPPORTING UBIQUITOUS WIRELESS COMMUNICATIONS}

It is well recognized that, to a certain extent, MC DS-CDMA constitutes a trade-off between SC DS-CDMA and MC-CDMA in the context of the system's architecture and performance. MC DS-CDMA typically requires lower chip rate spreading codes than SC DS-CDMA due to employing multiple subcarriers. It necessitates a lower number of subcarriers than MC-CDMA due to imposing DS spreading on each subcarrier's signal. Consequently, MC DS-CDMA typically requires lower-rate signal processing than SC DS-CDMA and has lower worst-case peakto-average power fluctuation than MC-CDMA. However, MC DS-CDMA goes far beyond constituting the trade-off between SC DS-CDMA and MC-CDMA. It exhibits a number of advantageous properties, which can be exploited to support ubiquitous broadband wireless communications in diverse propagation environments. Earlier we showed that MC DS-CDMA has the highest degrees of freedom in the family of CDMA schemes that can be beneficially exploited during the system design and reconfiguration procedures. Below we investigate how the specific parameters of MC DS-CDMA can be adjusted to satisfy the requirements of ubiquitous communications in diverse propagation environments.

The delay spreads are assumed to be limited to the range of $\left[T_{m}, T_{M}\right]$, where $T_{m}$ corresponds to the environment with the shortest delay spread considered (experienced, e.g., in an indoor environment). By contrast, $T_{M}$ is associated with an environment having the highest possible delay spread, as in an urban area. First, in order to ensure that the MC DS-CDMA system considered maintains the required constant frequency diversity order in different communication environments, the simplest approach is to configure the system such that each subcarrier signal is guaranteed to experience flat fading. Then the required constant frequency diversity gain is attained by combining the independently faded subcarrier signals, which is achieved with the aid of F-domain repetition or F-domain spreading. When communicating over various fading channels having delay spreads in the range of $\left[T_{m}, T_{M}\right]$, the flat fading condition of each subcarrier signal is satisfied if the chipduration, $T_{c}$, is higher than the highest delay spread, $T_{M}$ (i.e., when $T_{c}>T_{M}$ ).

Second, in order to achieve the highest possible grade of frequency diversity for a given Fdomain repetition depth, the subcarrier signals combined must experience independent Fdomain fading. This implies that the F-domain spacing between the combined subcarriers must be higher than the maximum coherence bandwidth of $(\Delta f)_{c M} \approx 1 / T_{m}$. Let $U$ be the number of data streams after the S-P conversion stage. Then the above condition is satisfied if

$$
\frac{U}{T_{c}} \geq \frac{1}{T_{m}},
$$

that is,

$$
U \geq \frac{T_{c}}{T_{m}},
$$

where $U / T_{c}$ is the minimum frequency spacing between two adjacent subcarriers conveying the same data bit.

The above philosophy might be augmented with the aid of an example. Let us assume that the total bandwidth of the broadband MC DSCDMA system is about $20 \mathrm{MHz}$. The delay spread is assumed to be limited to the range of $\left[T_{m}=0.1 \mu \mathrm{s}, T_{M}=3 \mu \mathrm{s}\right]$, which includes the typical delay spread values experienced in indoor, open rural, suburban, and urban areas. Based on the philosophy discussed earlier in this subsection, we can set $T_{c}=4 \mu \mathrm{s}>T_{M}=3 \mu \mathrm{s}$, and $U=T_{c} / T_{m}=4 \mu \mathrm{s} / 0.1 \mu \mathrm{s}=40$. When, for example, a total of 80 subcarriers occupying the spectrum of Fig. 3 and having a total system bandwidth of about $20 \mathrm{MHz}\left(=(80+1) / T_{c}\right)$ are employed, the repetition depth is two, that is, each data stream can be transmitted on two subcarriers. Consequently, this MC DS-CDMA system will operate efficiently over a wide range communication environments and will achieve a total diversity order of two, provided that the delay spread of the specific environment encountered is in the range of $[0.1 \mu \mathrm{s}, 3 \mu \mathrm{s}]$.
Future generations

of broadband

wireless systems

will aim to

support a wide

range of services

and bit rates in a

bandwidth on the

order of tens or

even hundreds of

megahertz. These

broadband

wireless signals

hence may have a

bandwidth

significantly

higher than the

coherence

bandwidth of the

channels

encountered. 
The Doppler

frequency shift of

the lowest and

highest subcarriers

may be substantially

different. This is

because broadband

MC DS-CDMA

may occupy a

system bandwidth

on the order of

tens or even

hundreds of $\mathrm{MHz}$.
Advantages - Based on the above rules for selecting the system parameters, broadband MC DS-CDMA is capable of mitigating the problems encountered by both SC DS-CDMA and MCCDMA. Specifically, broadband MC DS-CDMA has the following advantages:

-MC DS-CDMA is capable of supporting ubiquitous communications in environments as diverse as indoor, open rural, suburban, and urban areas. This is achieved by avoiding or at least mitigating the problems imposed by the different dispersion fading channels associated with the above-mentioned diverse communication environments.

- Broadband MC DS-CDMA guarantees that the combined subcarrier signals experience independent fading.

- Broadband MC DS-CDMA is capable of mitigating the requirements of high-chip-ratebased signal processing, as encountered in broadband SC DS-CDMA. This is achieved by introducing computationally efficient fast Fourier transform (FFT)-based parallel processing, carrying out modulation/demodulation for all subcarriers in a single FFT step. Broadband MC DS-CDMA is also capable of mitigating the worst-case peak-to-average power fluctuation experienced, since with the advent of using DS spreading of the subcarriers we have a significantly decreased number of subcarriers from MC-CDMA.

-In broadband MC DS-CDMA the orthogonality of the T-domain spreading codes remains unimpaired by fading-induced dispersion, since each subcarrier signal experiences flat fading. Therefore, for downlink transmission, users signatured using different $\mathrm{T}$-domain spreading codes can be detected with near single-user performance without employing MUD. The desired signal can be detected using conventional lowcomplexity single-user detectors.

-The achievable frequency diversity order is a constant value when communicating over a variety of fading channels. Consequently, when multiple BS antennas are employed, the transmit diversity scheme employed can be designed under the assumption of a constant frequency diversity order.

Disadvantages and Their Countermeasures With the aid of Table 1 we conclude that the two main deficiencies associated with broadband MC DS-CDMA are as follows.

- The Doppler frequency shift of the lowest and highest subcarriers may be substantially different. This is because broadband MC DSCDMA may occupy a system bandwidth on the order of tens or even hundreds of megahertz. The different Doppler frequency shifts corresponding to different subcarriers will destroy the orthogonality of the subcarriers, and a given subcarrier signal will experience intersubcarrier interference (ICI) imposed by the adjacent subcarrier signals. However, in most cases the ICI imposed by the other subcarrier signals becomes negligably low, provided that the mobile terminal's traveling speed is not too high. This is because the orthogonality between the desired subcarrier and its adjacent subcarriers remains relatively intact due to their similar frequencies.
By contrast, the distant subcarriers impose relatively low crosstalk on the desired subcarrier, since the crosstalk between the subcarriers decays inversely proportional to their F-domain separation [4].

-In broadband MC DS-CDMA each subcarrier signal experiences flat fading. According to the second column of Table 1 the achievable frequency diversity order also depends inversely proportionally on the number of parallel substreams. Hence, the frequency diversity order alone in broadband MC DS-CDMA may be insufficient for maintaining the required BER performance. However, the diversity order achieved can be augmented by using transmit diversity, as discussed in the context of $3 \mathrm{G}$ CDMA systems in [9].

\section{DS-CDMA USING TF-DOMAIN SPREADING}

In MC DS-CDMA using F-domain repetition it can be shown that for a given number of subcarriers $Q$, each having a constant chip rate of $R_{c}=$ $1 / T_{c}$, the number of bits per symbol, $U$, and the processing gain, $N=U T_{b} / T_{c}$, decrease upon increasing the repetition depth, $S$, due to the relationship of $Q=S \times U$. Hence, the number of users, $K$, supported by the conventional MC DS-CDMA using solely T-domain spreading also decreases upon increasing the repetition depth, $S$. This is because the number of DS spreading codes with good correlation characteristics is determined by the number of chips per symbol. Hence, for a given total system bandwidth, the maximum number of users supported and the frequency diversity gain achieved by using Fdomain repetition have to obey a trade-off. This is not a desirable result. We would like to achieve the maximum possible transmit diversity gain as well as the required frequency diversity gain without having to accept any further tradeoffs (i.e., without decreasing the total number of users supported by the system). This can be achieved by using both T-domain and F-domain (i.e., TF-domain) spreading in MC DS-CDMA systems.

The philosophy of the TF-domain spread broadband MC DS-CDMA system [1] may be augmented with the aid of Fig. 3 and Eq. 3. Specifically, as suggested by Fig. 3 and Eq. 3, each user in the system employs an F-domain spreading code in addition to a T-domain spreading code. Consequently, the total number of users supported by the TF-domain spread broadband MC DS-CDMA scheme is determined by the product of the T-domain spreading factor, $N$, and the F-domain spreading factor, $S$, that is, by $N \times S=\left(U T_{b} / T_{c}\right) \times(Q / U)=Q T_{b} / T_{c}$. Explicitly, the number of users supported by TFdomain spread MC DS-CDMA is independent of the frequency-diversity order $S$.

Spreading Codes Assignment - Let the maximum number of users that can be supported by the T-domain spreading codes be $K_{\max }$. It is well recognized that the number of $S$-length orthogonal codes used for F-domain spreading is $S$. Since the above two sets of orthogonal codes 
(the T-domain and F-domain codes) can be assigned to users independently, we can see that even if $S$ number of users share the same subset of T-domain spreading codes, these $S$ user signals might be distinguishable with the aid of the associated number $S$ of F-domain spreading codes. Hence, the spreading codes used in the broadband MC DS-CDMA system can be assigned as follows.

If the number of users is in the range of $0 \leq$ $K \leq K_{\max }$, these users will be assigned the required T-domain spreading codes and the same F-domain spreading code. However, when the number of users is in the range of $s K_{\max } \leq K$ $\leq(s+1) K_{\max }, s=1,2 \ldots, S-1$, the same subset of T-domain spreading codes must be assigned to $s$ or $(s+1)$ users, and hence these $s$ or $(s+$ 1) users have to be assigned different F-domain spreading codes. These $s$ or $(s+1)$ users employing the same subset of T-domain spreading codes are identified by their corresponding F-domain spreading codes.

Signal Detection - The subcarrier signals conveying the different chips of the F-domain spreading code encounter independent fading; therefore, the orthogonality of the orthogonal codes used for F-domain spreading cannot be retained. Hence, multiuser interference is inevitably introduced, which degrades the BER performance when increasing the number of users sharing the same subset of $\mathrm{T}$-domain orthogonal codes. However, in synchronous downlink broadband MC DS-CDMA using TFdomain spreading, the number of users sharing the same subset of T-domain orthogonal codes is only a fraction of the total number of users supported by the system, which assumes the maximum value of $S$. Hence, for this fraction of users advanced MUD algorithms [2] can be invoked in order to achieve enhanced BER performance while maintaining acceptable complexity. Accordingly, the receiver of broadband MC DSCDMA will have substantially lower complexity than that of broadband SC DS-CDMA or broadband MC-CDMA. Specifically, in the proposed system the MUD complexity depends on

$$
\left\lceil K / K_{\max }\right\rceil \text {, where }\lceil x\rceil
$$

represents the minimum integer lager than $x$, while in broadband SC DS-CDMA or broadband MC-CDMA the receiver's complexity depends on the total number of users supported, $K$.

Detection Performance - The BER vs. signal-to-noise ratio (SNR) per bit, $E_{b} / N_{0}$, and the performance of both the correlation-receiverbased single-user detector and the decorrelating MUD are shown in Fig. 4 for the TF-domain spread broadband MC DS-CDMA system. The parameters associated with Fig. 4 are: a total system bandwidth of $256 \mathrm{MHz}$, a single-user data rate of $R_{b}=1 \mathrm{mb} / \mathrm{s}, T_{x}=2$ transmitter antennas, length $N=32$ T-domain spreading codes, and length $S=8$ F-domain spreading codes, when supporting $K=1 \times 32,2 \times 32,3 \times 32$, and $4 \times 32$ users, corresponding to $K^{\prime}=1,2,3,4$ and $K_{\max }=32$. Note again that $K^{\prime}$ represents the

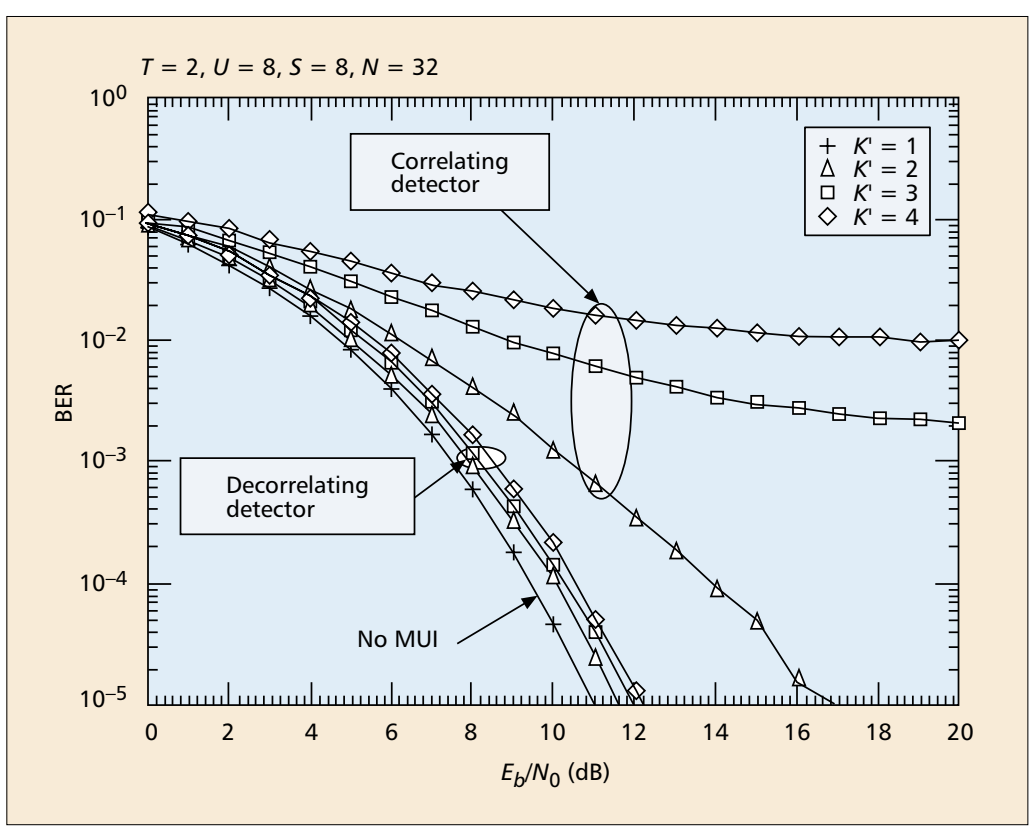

Figure 4. Simulation-based BER vs. the SNR per bit, $\mathrm{E}_{\mathrm{b}} / \mathrm{N}_{0}$, performance of both the single-user correlator and decorrelating MUD for transmit diversity assisted broadband MC DS-CDMA using BPSK modulation and TF-domain spreading when communicating over frequency-selective Rayleigh fading channels, where each combined subcarrier was independently flat faded.

number of users sharing the same subset of Tdomain spreading codes. From the results of Fig. 4 we observe that when using the decorrelating MUD, the SNR per bit of $E_{b} / N_{0}$ required to support $4 \times K_{\max }=128$ users increases only by about $1 \mathrm{~dB}$ over the scenario supporting $K_{\max }=32$ users.

\section{CONCLUSIONS}

The systems of interest studied in this article are broadband CDMA schemes supporting ubiquitous communications, regardless of the specific propagation environments encountered. Specifically, a broadband MC DS-CDMA was proposed for satisfying the requirements of broadband systems and supporting ubiquitous communications with the aid of transmit diversity. We have shown how the system parameters can be configured in order that the broadband MC DS-CDMA scheme becomes capable of supporting ubiquitous communications without compromising the achievable BER. We have also considered the user capacity improvement in broadband MC DS-CDMA with the aid of TF-domain spreading. Our studies suggest that broadband MC DS-CDMA using transmit diversity constitutes a promising multiple access scheme capable of avoiding the various design limitations encountered when communicating over diverse propagation environments. For more details on the associated schemes please refer to [1].

\section{REFERENCES}

[1] L. Hanzo et al., Single- and Multi-Carrier DS-CDMA Multi-User Detection, Space-Time Spreading, Synchronisation, Standards and Networking, IEEE Press-Wiley, June 2003, $1077 \mathrm{pp}$. (for sample chapters go to http:// www-mobile.ecs.soton.ac.uk) 
Broadband MC

DS-CDMA using

transmit diversity is

a promising

multiple-access

scheme capable

of avoiding the

various design

limitations

encountered when

communicating

over diverse

propagation

environments.
[2] M. Progler, C. Evci, and M. Umehira, "Air interface access schemes forbroadband mobile systems," IEEE Commun. Mag., Sept. 1999, pp. 106-15.

[3] R. Prasad and S. Hara, "Overview of Multicarrier CDMA," IEEECommun. Mag., Dec. 1997, pp. 126-33.

[4] L.-L. Yang and L. Hanzo, "Performance of Generalized Multicarrier DS-CDMAover Nakagami-m Fading Channels," IEEE Trans. Commun., vol. 50, June 2002, pp. 956-66.

[5] E. A. Sourour and M. Nakagawa, "Performance of Orthogonal Multicarrier CDMAin a Multipath Fading Channel," IEEE Trans. Commun., vol. 44, Mar. 1996, pp. 356-67.

[6] L. Vandendorpe, "Multitone Spread Spectrum Multiple Access Communications System in a Multipath Rician Fading Channel," IEEE Trans. Vehic. Tech., vol. 44, no. 2, 1995, pp. 327-37.

[7] W. C. Y. Lee, Mobile Communications Engineering, New York: McGraw-Hill, 2nd Ed., 1998.

[8] V. Tarokh and H. Jafarkhani, "On the Computation and Reduction of the Peak-to-Average Power Ratio in Multicarrier Communications," IEEE Trans. Commun., vol. 48, Jan. 2000, pp. 37-44.

[9] R. T. Derryberry et al., "Transmit Diversity in 3G CDMA Systems," IEEE Commun. Mag., vol. 40, Apr. 2002, pp. 68-75.

\section{BIOGRAPHIES}

LIE-LIANG YANG [SM] (Ily@ecs.soton.ac.uk) received his M.Eng. and Ph.D. degrees in communications and electronics from Northern Jiaotong University, Beijing, China, in 1991 and 1997, respectively, and his B.Eng. degree in communications engineering from Shanghai TieDao University, China, in 1988. Since December 1997 he has been with the Communications Group, Department of Electronics and
Computer Science, University of Southampton, United Kingdom, and currently holds an academic post as a lecturer. His research interests are in the areas of error correction coding, modulation, and detection techniques, as well as wideband, broadband, and ultra wideband CDMA systems for advanced wireless mobile communication systems. He has published over 80 research papers in journals and conference proceedings.

LAJOS HANZO [SM] (Ih@ecs.soton.ac.uk) received his Master's degree in electronics in 1976 and his doctorate in 1983. During his 26-year career in telecommunications he has held various research and academic posts in Hungary, Germany, and the United Kingdom. Since 1986 he has been with the Department of Electronics and Computer Science, University of Southampton, United Kingdom, where he holds the chair in telecommunications. He has co-authored 10 books totaling 8000 pages on mobile radio communications, published about 400 research papers, organized and chaired conference sessions, presented overview lectures, and been awarded a number of distinctions. Currently he leads an academic research team working on a range of research projects in the field of wireless multimedia communications sponsored by industry, the U.K. Engineering and Physical Sciences Research Council (EPSRC), the European IST Programme, and the Mobile Virtual Centre of Excellence (VCE), United Kingdom. He is an enthusiastic supporter of industrial and academic liaison and offers a range of industrial courses. He is also an IEEE Distinguished Lecturer. For further information on research in progress and associated publications please refer to http://www-mobile.ecs. soton.ac.uk. 